\title{
Was the Internet the Only Option? - Which Way Should Business and Information Systems Engineering Go?
}

\author{
In the same way as production methods or dominant materials have been representative \\ for entire eras so far, important parts of present-day life can indeed be identified with the \\ Internet. Multimedia-based communication results from digitalization, and the almost \\ cambrian-like explosion of end-user devices and services revolutionized the organization \\ of everyone's work and leisure. After the internationalization of telecommunication, \\ which was organized nationally up to the 1990s, now standardization of business and \\ private communcation and organization is under consideration. The article reviews \\ the development of the Internet in order to gain a better view on the proceeding \\ development by affirming the past. The analogy to problems and questions of business and \\ information systems engineering (BISE) establishes the reference to present-day issues.
}

DOI 10.1007/s12599-008-0030-3

\section{The Author}

Prof. Dr. Günter Müller

Institute of Computer Science and

Social Studies, Department of

Telematics

University of Freiburg

Friedrichstr. 50

79098 Freiburg

Germany

mueller@iig.uni-freiburg.de

Received: 2008-05-04

Accepted: 2008-07-31

Accepted after three revisions by

Prof. Dr. Buhl.

This article is also available in German in print and via http://www.wirtschaftsinformatik.de: Müller G (2008) War Internet die einzige Option? - Welchen Weg soll die Wirtschaftsinformatik gehen? WIRTSCHAFTSINFORMATIK. doi: 10.1007/11576-008-0140-z.

\section{Introduction}

The 1980s and 1990s constitute the decisive period when the Internet advanced to its present-day hegemonic position. The existent proprietary networks of all IT-providers, foremost IBM with the SNA (Systems Network Architecture) which was widely spread at that time, and the open architecture of the European OSI (Open Systems Interconnection), seemed to hinder the development of the more academically-oriented Internet - then known under its former name ARPAnet. The position of the Internet can only be considered to be all-dominant since its commercial use, i. e. after the shutdown of ARPAnet in 1990.

The $50^{\text {th }}$ anniversary of the WIRTSCHAFTSINFORMATIK journal not only conduces to a proud and critical view back to the past but also mainly to the discussion of BISE's future. Therefore, this article about the Internet does not constitute an editorial mistake but intends to dare a glance at current research strategies in BISE and Information Systems (IS) research respectively. This is done by recapitulating the key success enablers of the Internet. Today, there are different confronting ideologies as regards BISE - just as it was in the past as regards the Internet.

The following disquisition is structured into four sections. At first, the possibilities of former telecommunication as well as of present BISE will be outlined. The second and the third part then concentrate on the Internet and describe the position of the disputing parties as well as the events and decisions during the so-called "wild phase" between 1987 and 1992. This will be done from the perspective of IBM's European Networking Center in Heidelberg (Müller 1990). The fourth part considers "looking ahead" and discusses possible research strategies and whether a promising dominance of a specific approach for BISE can be concluded from "looking back".

\section{Initial status of communication and informatization}

When computers had to be coupled across organizational units before 1985 , only PTT networks (postal, telephone, telegraph) could be used, even if there were cheaper possible solutions. Especially the high costs of telecommunication, which were considered to be technologically unjustified, and applications limited to speech and fax showed the "backwardness" of the PTTs, which thus became obvious and led to proposals for a deregulation among other things. This authorized first end user devices and also since 1988 networks for competition. By means of VoIP (Voice over 
IP), a total decrease of the PTT's network and speech monopoly is to be expected. Then, the dominance of the Internet as the only option of telecommunication will be complete.

Today, informatization is the way of thinking as was technological communication in the past. Until the end of the 1980s, communication was conceived as installing data centers. Not before the "unbundling" carried out by the American antitrust offices in the late 1970s, which was primarily directed against IBM, could the application programs' "Babylonian captivity" of hardware and system software be dissolved. Information systems became separated from their physical mediums. BISE's object of research - information systems - took its present-day form in a stepwise manner and thus enabled competition for a dominant approach.

\subsection{Controversy on directions in BISE}

Since the publication of Nunamaker et al. (1991) at the latest, different research strategies in BISE are considered as a controversy over directions or methods. On the one side there are researchers of the socalled IS-branch (Information Systems), who are supporters of an "empirically oriented behaviorism". On the other side there are those researchers who consider BISE as part of the "design sciences". Whereas in the USA and also often in the journals behaviorism dominates, "design science" with its traditions positioned in engineering sciences and business economics takes a predominant role in the German-speaking countries (Bichler 2006). There, computer science methods are used to solve business problems. IS research dominated by behavioral science perspectives does not focus on IT itself but tends to deal with its implications for individuals, groups, and organizations. Opposed to that, the objective of design science-oriented BISE research is marked by creating and evaluating IT-artifacts with respect to their utilization for information systems (overview in Frank 2003). "The behavioural-science paradigm relies on truth, the discovery of truth. In contrast, the design-science paradigm seeks to create what is effective" (Hevner et al. 2004).

\subsection{Controversy on directions in telecommunications}

Thirty years ago, the situation within the telecommunication sector was even more heterogeneous as it is in BISE today. It was intended to create a global infrastructure in order to unify "computers" and "communication". In those days this was primarily conceived as cooperation between PTTs and IT-providers. However, the situation of today was unpredictable in the same way as it is surprising. It consists of an internationalization of the thitherto nationally organized telecommunication and a reorganization of the entire economic infrastructure as a consequence of digitalizing all mediums. The protagonists involved did not even agree on how to define a computer network. While IT-providers primarily connected mainframe computers and aimed at surviving in competition with their proprietary applications, the PTTs feared to lose their monopoly in speech communication. The third party - represented by the Internet - demanded an end-to-end communication by creating an open platform in order to enable all users to create their applications autonomously.

\subsection{Telecommunication policy of IBM and the "mission" of ENC Heidelberg}

In 1980, IBM found itself for the first time in the position that the company was not able to define an important IT-standard on its own. The heterogeneity of the coalitions due to incompatible objectives enabled neither IBM nor its competitors or the political entities (e.g. the European Union) to formulate a consistent telecommunication policy. Everybody agreed that the upcoming replacement of analog by digital technology and the inevitably resulting reorganization of the communication market had to be settled in a controversial and costly discourse. Here solutions could coexist until finally a market-driven answer for the infrastructure-related developments was reached. The European Networking Center (ENC) was founded in 1985 as a technologicalscientific "branch" of IBM to take part in this technical discourse.

The following four questions cover the ENC's objectives without including speech communication and the PTT's network monopoly:
- Global infrastructure: Is it intended to have a global and unified network architecture for all forms of communication or should proprietary networks persist?

- End-to-end: Is it intended to connect computers only and/or applications as well? What might be a possible "killer application"?

- Network architecture: Is OSI the "lingua franca" or is it just another network architecture?

- Network functionality: Should standardization include applications as well as data communication?

The way to go was a cooperation with the European IT industry (club of the twelve) and politics. All of these organizations favored the emerging European telecommunication standard OSI (Open Systems Interconnection) and supported it by means of national projects; this also happened in Germany with the still existent DFN (German research network). However, this technological policy was too abstract and often provided the justification for speaking of "muddling through" or "ideologically" motivated "skirmishes" for a lot of participants.

\section{Competing network models}

The competing proposals and strategies differed with regards to the network model (Cypser 1991), owner rights, treatment of applications, and the way in which networks are to be run and standards are to be reached. Carpenter summarized the difference of the Internet to the competing options in his RFC 1958 (Request for Comments): "in very general terms, the community believes that the goal is connectivity, the tool is the Internet-protocol, and the intelligence is end-to-end rather than hidden in the network" (Carpenter 1996).

\subsection{Public Switched Telephone Network}

The PTTs were aware of the fact that without employing great efforts they would emerge as losers in the digital age. Their concepts as regards data communication were insufficient and the monopoly in speech communication had been put at risk for the short term in the U.S. due to the discussions on deregulation, but also in Europe for the long term. 
POTS (Plain Old Telephone Service) was a nickname of the Public Switched Telephone Network (PSTN). This term also fits the newer digitalized telephone networks that were enriched with further service characteristics. Consequently, all services of the analog telephone services could be described. Concerning network functionality, the PTTs did not aim at separating applications from communication. This does not surprise considering that PTTs did not live from the networks themselves but from using them. The high efforts of Minitel in France and Bildschirmtext (BTX) in Germany constitute conclusive evidence for the seriousness with which this "connected" business model was also pursued within data communication as well.

Fax and speech were the POTS' only important applications. Other services such as telex - had their own networks, which however were not used for PSTNconnections. In 1980, these parallel subnetworks led, together with digitalizing routers and signal transmission, to an "integrated services digital network", the ISDN. In Germany public telephone networks have stopped using analog telephone networks since the 1990s.

\subsection{Systems Network Architecture (SNA)}

SNA was introduced by IBM in 1974 and is partly still in use today. The digital network architectures of IBM and its competitors, which were summarized disrespectfully under the term BUNCH (Burroughs, Univac, NCR, Control Data, Honeywell), have dominated the IT-market in a monopolistic way since 1980 . They were conceptionally identical and usually only had an intra-organizational range of application. This limitation was not an outcome of technological reasons but resulted from the PTTs' high communication costs. The consequence was that all computer networks connected "stupid terminals" with mainframes and that other perspectives were difficult to place with IT-providers for a long time.

SNA had been developed to support "host-to-terminal" communication but additionally realized peer-to-peer networks since 1985 . The system software, the VTAM (Virtual Telecommunications Access Method), is the programming interface on a mainframe computer. Only the NCP (Network Control Program) on the front end computers establish a con- nection to the hard-wired monitor- and printer-terminals. Connections (sessions) are established and released between routers in the network only. Communicating with more than one user has been possible since the introduction of the so-called LU (Logical Unit) in the beginning of the 1980s, where each LU can establish exactly one connection to an application program on a mainframe computer. By means of the upcoming "intelligent end-user devices" and the then foreseeable expiring of "stupid terminals", the LUs' functionality had been increasingly replaced by software solutions that on the one hand emulated the former terminals and on the other hand enabled the parallel dial-up to multiple application programs.

The limitation to mainframes hindered the further development of SNA and raised the belief that not only the PTTs but also IBM “overslept" the technological development and the already forseeable triumph of PCs (Personal Computer).

\subsection{Open Systems Interconnection (OSI)}

The OSI reference model had been developed since 1978 by the ISO (International Standards Organisation) and the PTTs with the objective to create an architecture being able to serve as a guideline for supporting computer-to-computer communication. The ISO-OSI reference model was passed in 1982 and consists of two parts: Protocols on the one hand and service descriptions on the other. It constitutes a hierarchical network model that separates communication into seven layers. The main criteria for this delimitation are the service units. The number of layers should be sufficiently high to find all functions exactly once (des Jardins 1981). By means of this modularization it was possible to modify or exchange implementations without affecting other layers (Müller et al. 2003a).

The upcoming digitalized audio- and visual media (multimedia) extended the range of application of the OSI-model significantly, as it happened again later with the upcoming of personal computers and mini-computers. Processing and storage power of the systems at that time were still too limited for higher requirements. Only in the mid-eighties was it possible to meet this challenge with ODP (Open Distributed Processing), which among others was developed within the project HECTOR (Heterogeneous Computers Together) at
Karlsruhe University in cooperation with the ENC.

The formal brilliance of the OSI reference model also constituted its weakest point. Interoperability was only possible if the communication software was totally conform. For this purpose OSI-profiles were defined, which proved to mainly enforce the pressure of standardization. The call for a "killer application" to cover the significant infrastructure-related costs was understandable but turned out false in the end. The Internet never had such an ambition nor this pressure. Right from the start TCP/IP considered applications as a matter of users who would be more qualified and who should only be provided with a platform to exchange applications.

\subsection{Internet (TCP/IP)}

Adventurous stories abound about the origin of the Internet. For instance, there is a very popular version maintaining that the Internet's predecessor ARPAnet had been installed to protect the U.S. military mainframe computers against nuclear attacks. This explained the use of packet switching instead of circuit switching - the latter was usually used with the POTS, the interplay of different networks, and the abstinence of applications. Reality is much more civil.

In 1968, ARPA (Advanced Projects Research Agency) announced an experiment that was indeed challenging at that time. Four mainframe computers in the Western USA were supposed to be connected. IBM refused to participate due to the project's economic inefficiency from their point of view. Surprisingly, Bold, Beranek \& Newman (BBN), a counseling company for architectural acoustics, was accepted. In December 1969, a functioning network between the Stanford Research Institute and the Universities of Santa Barbara, Los Angeles, and Salt Lake City based on IMP (Interface Message Processor) could be demonstrated. Here, packet switching was used. Each IMP, called router in later times, divides the data to be sent into small packets and adds the recipient's and the sender's addresses. By means of routing tables these packets can be transmitted even redundantly via available circuits from computer to computer without depending on a total overview of all routes. Having arrived at the recipient's computer the packets are assembled into their original order. 
The two technological break-throughs described next particularly changed the previous logic which was based on the opinion that the amount of communication cost depends on the PTTs and thus is unalterable. In 1968, ALOHAnet evolved as a radio- and packet switching-based communication form which connected the mainframe computers on the islands of Hawaii in a cheap way. This was also because the PTTs could be avoided (Kahn and Cerf 1999) by being too innovative. The technical break-through was an approach to avoid collisions. A couple of years later, the idea of temporally delayed transmission of packets made its way to local communication. Bob Metcalfe showed that the packet principle and collision avoidance also worked with cables (Metcalfe 1973). The Ethernet caused an explosive expansion of local networks primarily because it allowed the reduction of communication costs to almost zero (Cerf and Kahn 1974).

Moreover, by introducing the ALOHAnet a third important principle became reality, the influence of which, however, was not comparable to the other two break-throughs mentioned before. Networks should not be connected at the network layer except when the functionally weakest network is supposed to determine the range of services. The illusion of the Internet as a homogenous network from user perspective could only be sustained at the transport layer because there it was possible to control an end-to-end chain of sub-connections by using a simple transmission control program. Only in 1978, Cohen and Postel carried out the division of TCP and IP (Postel 1980) and thus set today's effective standard of the Internet (David and Shurmer 1996), which propagates a more social than technological guideline (Saltzer et al. 1984) by means of "freedom of action, user empowerment, end-user responsibility, and lack of controls in the net, that limit or regulate what user can do" (Blumenthal and Clark 2001).

The competitors' insistence on an interconnection of applications and communication offered the strategic gap which only had to be taken on by the Internet. In this respect, the telecommunication policy of the PTTs and the IT industry appears from a retrospective view - incomprehensively far away from technology.

\section{The "wild phase" of competition between 1987 and 1992}

Since 1985, IBM was able to offer its own end-to-end solution with the "Advanced Peer-to-Peer Communication”. Additionally, they were willing to publish all proprietary SNA protocols as it was claimed by the EU and the American anti-trust office. SNA could have become the blueprint for a global telecommunication infrastructure. However, at no point of time was IBM able to gain the trust of ISO, of the PTTs, or even of all of their IT-competitors and of politics.

\subsection{The dual strategy of IBM and the Department of Defense}

Nevertheless, IBM participated - to the surprise of many - in projects that were clearly opposed to their own product announcements or strategies. Together with the most important proponents of OSI worldwide they designed and realized a functioning network with an OSI-architecture for a demonstration at TELECOM 1987.

\subsubsection{TELECOM 1987: OSI as the apparent winner}

Every four years the PTTs presented their technological innovations and options at a fair in Geneva. Out of these the technological innovations for the telecommunication market were determined and standardized by the CCITT (Commité Consultatif Internationale Télégrafique et Téléfonique).

IBM pursued three objectives at TELECOM:

1. OSI should connect the proprietary worlds of IBM and BUNCH and demonstrate its usefulness "as a network model between networks".

2. Reducing OSI to a technology "between the networks" should oblige the PTTs to extend their infrastructure more quickly, which was supposed to charge off quickly due to increasing demand and to reduced communication costs.

3. The worldwide participation of the most important PTTs and the major ITproviders should accelerate the restriction of OSI to a "network between networks".

None of these objectives - except for a "gateway" for message and data transfer based on the p5/2 protocol - could be realized (Müller and Schulze 1985). In contrast, under the pressure of the OSI consortium the first large OSI-network evolved that confirmed OSI as an independent network architecture. Remarkably, the guidelines and procedures for "Networking in Open Systems" with OSI had already been collected and discussed by IBM and the NBA (National Bureau of Standards), called NIST (National Institute of Standards and Technology) today, at a conference in Oberlech, Austria (Müller and Blanc 1987). Operations at the Tokyo train station with its impressive Shinkansen bullet trains could be observed in realtime from Geneva in the same way as the advertisements at New York Times Square or the ringing of Big Ben in London. All in all more than 30 enterprises worldwide participated in this demonstration. The double role of IBM and the "non-action agreement" of the participants seemed to pay off because the "relevant" offices of the DoD (Department of Defense) could be convinced of OSI. Electronic mail with X400 , gateway $\mathrm{p} 5 / 2$ for data transfer, remote access to databases, and the possibility of multimedia constituted a solid basis for developing the generally acknowledged services from that point on.

Except for IBM also the American Department of Defense took a Janusfaced double role because it continued to support the Internet but preferred OSI for military procurement (Witt 1986). The evaluation of TP-4 (transport protocol of OSI) and TCP/IP carried out in 1983 led the DoD to the conclusion to pursue both options simultaneously for the time being, but to require TP-4 for military use (Postel 1985). This became manifest in 1988 when the OSI variant GOSIP (Government Open Systems Interconnection Profile) had been declared as standard (Cerf and Mills 1990) and in 1990 when all American offices were supposed to buy communication products based on GOSIP only (Caffrey 1990). However, due to the fact that using other communication networks had not been prohibited simultaneously mostly network administrators opposed this requirement and continued running their networks according to TCP/ IP (Huizer 1993).

\subsubsection{Post-TELECOM 1987}

IBM's inconsistent technology policy became obvious by four subsequent announcements: 
1. GOSIP had been declared as standard for the DoD and thus became the hope of OSI (Hart 2004).

2. Two years before (1985) advanced peerto-peer SNA had been announced as a piece of impressive technological progress which was bound to supersede OSI for all of IBM's customers, or in the best case could present SNA superior to OSI.

3. The ENC was put in charge of EARN (European Academic Research Network). EARN was a scientific initiative of IBM and granted free access to computer networks for European universities. In agreement with DFN a possible later replacement of the proprietary IBM-protocols by OSI was announced (Schulze and Müller 1985).

4. Surprisingly, OSI and the Internet agreed on working together on a new and now common network protocol as compensation for IP in 1987.

The success of OSI influenced the Internet. It was now possible to cooperate with OSI which was hoped to generate a solution of the addressing problem caused by IPv4 (Huitema 1998).

\section{2 "Constitutional" crisis of the Internet}

IP was supposed to be replaced by CLNP (Connectionless Network Protocol). The acceptance of the already tested CLNP failed in Kobe, Japan in 1992 due to the members of IETF (Internet Engineering Task Force). With their proposal of CLNP, Vinton Cerf und David Clark committed a "betrayal" (Blumenthal and Clark 2001) which hit "angry" and "furious" programmers who were not willing to "make peace" with OSI. Cargill talked about a "constitutional crisis", which could only be settled after the "Fathers of the Internet" publicly and impressively had confessed their "mistake" (Cargill 1997).

For IBM many wishes would have become true with CLNP. It would have had justified the unclear technology policy afterwards because this would have confirmed the restriction of OSI to the "gateway function". The Internet community assumed a "foul play" by the ITproviders, the PTTs, ISO, and the military to avoid an infrastructure that is unified, low-cost, and above all accessible for everyone. The statement "TCP/IP is suitable for technology, while OSI is suitable for theology" (Padlipsky 1985) only rudimentarily shows the prevalent emotional- ism and rejection of any hierarchy. Richard des Jardins, president of NBA, noted wearily: "these are people who object to the convergence of cultures and races of the world at large" (des Jardins 1992). OSI appeared as a concept of a regularized world of economy, military, and ISO. On the contrary, the Internet opened a wider space for innovation as the lawyer Lessing started ten years later (Lessig 2001).

Cerf and Clark mastered this crisis in a theatrical manner after Huitema (Blumenthal and Clark 2001), who co-developed CLNP (Huitema 1998), had failed with his explanations in front of an auditorium of technicians. Vinton Cerf symbolically returned to the circle of programmers by getting rid of the jacket and the vest of his three-part business suit during his apology and visually acknowledging his "heresy" in habitus and content by revealing his T-Shirt with the inscription "IP". David Clark coined the following motto that made an enormous impact on this meeting: "We reject kings, presidents, and voting. We believe in rough consensus and running code" (Clark 1992).

As a consequence of IBM's and the DoD's dual strategy and the unchangeable position of IETF, the OSI community lost momentum and SNA could be considered to have failed socially. Until 1995 - after the upcoming of the World Wide Web (WWW) (Berners-Lee 1999) - there was a kind of "ceasefire" between the remaining parallel worlds OSI and Internet. From 1992 on, IBM got into serious economic difficulties and made no attempt to dominate the network world from that time on. The ENC in Heidelberg was dissolved in 1995 after the WWW's success and after an eventful decade in the course of a worldwide reorganization of IBM.

\section{Internet: From the option to hegemony - Analogies to the future of BISE?}

During the decade from 1985 until 1995 the Internet with its TCP/IP, which we regard as a matter-of-course today, evolved as the winner from a fiercely conducted technological-scientific quarrel. This discourse has changed the world. Telecommunication is deregulated, the dominance of IBM is broken, the variety of IT-infrastructures is reduced to a few concepts in hard- and software, access to the Internet is equivalent to participating in the progress that impressively showed its power with the WWW and dominated the second half of the 1990s. Further innovations that have been caused by the Internet are to come when you look at the concepts discussed in connection with the catchword Web 2.0 at the moment.

Even today, we applaud Vinton Cerf and Robert Kahn for their technological contributions. Just as with the other Internet pioneers, one forgets that they reached their "position among the gods" more by creating institutions, outstanding management, and good judgment. In retrospect, there was no point of time when OSI or SNA really had a chance for being successful. In this context, Drake accurately stated: "The debate is not about the comparative efficacy of two sets of standards, but is rather between two competing visions of how international standards and network development should be organized and controlled" (Drake 1993). The visions of SNA and OSI were not stable enough to weave the future of IT:

1. Especially today it is hardly imaginable that political institutions would have had put the "nervous system" for the emerging information society exclusively into the hands of IBM.

2. The Internet contrasted the technological advantages of OSI and SNA for a central error reporting and for a locally optimized network topology with integration of technological progress, user friendliness as well as openness and transparency.

3. Response times of IETF easily surpassed ISO by far so that one can talk about a "hare- and hedgehog-race". Up to today decisions for the Internet and the WWW never take longer than 18 months, whereas ISDN in comparison only became marketable after more than 35 years of standardization.

4. However, the chance on a merger between the Internet and OSI that had been given away in 1992 would have at least partly avoided the obvious disadvantages and risks as regards security and protection of privacy (Müller 2008).

What does this review reveal about future research strategies of BISE, and may it even hint at a successful dominance of a specific approach? The only thing that is certain is that BISE must continue considering the development of IT as one of its major interests and has to participate in the evolving "third phase" of Internet 
usage. Especially IBM and the other ITproviders, but also the PTTs failed among other things due to their misjudgment of this development process.

The following text first gives an outline of the analogies between BISE and the Internet based on the "Design Guidelines" by Hevner (Hevner et al. 2004). Finally, the difference between a science - like BISE and systems engineering - like the Internet - is elaborated in particular:

1. Design as an artifact: By means of TCP/ IP and the vision of "connecting", an artifact "Internet" has been created that is able to solve a problem better than the competing network models.

2. Problem relevance: The requirements were not set but collected in an iterative way with participation of the users. The actual problem did not consist in identifying a killer application but in "connectivity" and creating a platform enabling innovation.

3. Design evaluation: Conceptional and model-oriented thinking is important but remains ineffective without evaluation. Functional systems were and still are today the accepted and necessary proof of every concept with the "Open Systems Community" and the WWWconsortium, as they are with all other standards as well.

4. Research contributions: Packet switching, the creation of a unified namespace, usage of radio-based communication, the extremely innovative business concept, and the integration of new IT end-user devices constitute milestones in research. Usability of the platform now, with the Web 2.0 following the WWW, proves to be flexible enough to enable new services.

5. Design as a search process: The Internet evolved by a perennial process for which new organizational forms and institutions were created that are still used today, e. g. in the WWW. In the Open Source Community they display a strength that they made them become the only serious competitor for Microsoft and its vision of the future of IT.

6. Communication of research: The Internet has not been conveyed to the management of established sciences, industry or politics, but has procured an allegiance within the academic field of those keen on making experiments.

Criticism on the design science-oriented approach of BISE (discussion in Frank
2003) has been raised in the same wording for the Internet (des Jardins 1992):

1. It is complained that the Internet's low level of theoretical foundation is suitable for one-time break-through but not in the long run.

2. The number of constructs, models, methods, and tools is not actually new and a one-time activity which is not transferable was not suitable for generalization.

3. The subordination of Internet institutions is said to become obvious sooner or later as technology progresses.

As convincing as this analogy seems, the fact that BISE is a science and the Internet is the result of systems engineering is not to be ignored. Sciences are determined by their object of research and do not constitute a result of development.

- Research results of BISE are artifacts. Artifacts are divided into constructs, models, and methods (Bichler 2006). BISE is determined by its object of research and not by a however defined achievement of objectives.

- BISE as all other sciences is judged from the innovations it performs in its field. In the case of the Internet such a claim would be totally inappropriate because here only the service performed by the Internet tool counts.

- Systems engineering depends on guidance and clearly set objectives and milestones. A young science like BISE is rather fruitless without competition. Therefore, heterogeneity, which has been lamented in places, should not be interpreted as a bad thing but rather as a sign of vitality.

Often the development of an idiosyncratic theory is demanded in a kind of reflex which would then certainly lead to a roadmap. The example of the Internet impressively shows that this is an unnecessary constriction. Acting according to one theory would definitely have established SNA as the network model dominating at that time. Simon's variety of methods (Simon 1996) seems to better meet the requirements of a young discipline because he views the competition for the best method as a "struggle" for the "right" solution. Today and in future such an integrative perspective provides BISE with a reputation that the engineering sciences have finally reached after a decade-long dispute over their scientific status. Instead of looking for possible delimitations from the neighboring disciplines one should emphasize similarities more strongly, especially as suspicion exists that a strict separation according to methods tends to originate from the politically motivated way of thinking in disciplines characteristic of the academic world.

In my opinion, a shift of the current majority within the American IS community towards design science can be expected because challenges of BISE will increase. Then "useable" solutions will count which are unlikely to arise without considering IT development. Future computer forms, e. g. Ubiquitous Computing, make other demands on the design methods, security in service-oriented architectures is totally unclear, and there is the risk of considerably neglecting privacy (Müller et al. 2003b).

BISE is faced with numerous questions that come along with technological progress and require new or changed organizational and cooperational forms. Only their interplay enables a modern network economy. The question is which direction of BISE will make "relevant" contributions to informatization in the future (Mertens 1995) - among other things initiated by the WWW and now continued with the cooperative communication forms of the Web 2.0. The answer will be given by the party able to think of and develop innovative contributions for solving present and future problems of the information society.

\section{References}

Berners-Lee T (1999) Weaving the web: the original design and ultimate destiny of the world wide web by its inventor. Harper, San Francisco, pp $16 \mathrm{ff}$

Bichler M (2006) Design science in information systems research. WIRTSCHAFTSINFORMATIK 48(2):133-142

Blumenthal M, Clark D (2001) Rethinking the design of internet. ACM Trans on Internet Technology 1(1):70-109

Caffrey L (1990) EPHOS: towards a european GOSIP. Computer Networks and ISDN Systems 19(3-5):265-269

Cargill K (1997) Open systems standardization: a business approach. Paladin Consulting

Carpenter B (1996) Architectural principles of the internet, IETF RFC 1958. http://www.ietf.org/ rfc/rfc1958.txt. Accessed 2008-08-01

Cerf VF, Kahn RE (1974) A protocol for packet network intercommunication. IEEE Trans Comm 22(5):637-648

Cerf VF, Mills K (1990) Explaining the role of GOSIP. IETF RFC 1169. http://www.ietf.org/rfc/ rfc1169.txt. Accessed 2008-08-01 


\section{Abstract \\ Günter Müller \\ Was the Internet the Only Option? - Which Way Should Business and Information Systems Engineering Go?}

In global competition, the Internet turned out to be the single and hegemonial infrastructure for communication. It has become the "nervous system" of today's networked economy. While the first phase provided communication services, like e-mail, the WWW has established an interactive platform to allow easy access to advanced services. Now, in its "third" or cooperative phase, the Internet will finally lead to a ubiquitous informatization where business processes and applications become interleaved beyond the boundaries of enterprises. For this phase, many analogies to the emergence of the Internet can be observed.

Keywords: Internet, Network models, OSI, SNA, ISO, IETF, Open source community, Standardization, Network institutions, Regulation, Network services, Behavioral vs. design science-oriented approach in BISE and IS
Clark D (1988) The design philosophy of the DARPA internet protocols. Proc. SIGCOMM 88. Computer Communication Rev. 18(4):106-107

Clark D (1992) A cloudy crystal ball: visions of the future. Proceedings of the $24^{\text {th }}$ internet engineering task force. The Internet Society

Cypser RJ (1991) Communications for cooperating systems, OSI, SNA and TCP/IP. AddisonWesley

David PA, Shurmer M (1996) Formal standardssetting for global telecommunications and information services. Telecommunications Policy, pp 789-815

des Jardins R (1981) Overview and status of the ISO reference model of open systems interconnection. Computer Networks 5(2):77-80

des Jardins R (1992) OSI is (still) a good idea. ConneXions 6(6):33

Drake W (1993) The internet religious wars. Telecommunications Policy, p 643

Frank U (2003) IS research relevance revisited: subtle accomplishment, unfulfilled promise or serial hypocrisy? WIRTSCHAFTSINFORMATIK 45(3):354-357

Hart D (2004) Corporate technological capabilities and the state: a dynamic historical interaction. In: Lipartito K, Sicilia DB (eds) Constructing Corporate America: Historical Perspectives on Big Business, Society, and Politics. Oxford Univ. Press, pp 168-187

Hevner AR, March ST, Park J, Ram S (2004) Design science in information systems research. MIS Quarterly 28(1):75-105

Huitema C (1998) IPv6: the new internet protocol. Prentice Hall PTR. pp $2 \mathrm{ff}$

Huizer E (1993) The IETF integrates OSI related work. ConneXions 7(6):26-28

Kahn RE, Cerf VG (1999) What is the internet (and what makes it work). http://www.cnri.reston. va.us/what_is_internet.html. Accessed 200805-01

Lessig L (2001) The future of ideas: the fate of the commons in a connected world. Random House

Mertens P (1995) Wirtschaftsinformatik - Von den Moden zum Trend. In: König W (ed) Jahrestagung der Wirtschaftsinformatik ‘ 95 , Frankfurt

Metcalfe RM (1973) Packet communication. MIT project MAC technical report MAC TR-114

Müller G (1990) IBM European Network Center annual report 1990. TR 45.9001

Müller G (2008) Abschlussbericht zum Schwerpunktprogramm Sicherheit der DFG. http:// www.telematik.uni-freiburg.de/spps/abschlussbericht.php. Accessed 2008-08-02

Müller G, Schulze G (1985) Internetting on the message handling layer. IFIP 2nd international symposium on computer message systems, Washington

Müller G, Blanc RP (1987) Networking in open systems. Springer, Heidelberg

Müller G, Krüger G (1988) LAN and OSI based heterogeneous campusnetworks. In: Krüger $G$, Müller G (eds) Hector, vol II. Springer, Heidelberg, pp 11-30

Müller G, Kreutzer M, Eymann T (2003a) Telematik- und Kommunikationssysteme. Olden- bourg, München

Müller G, Kreutzer M, Strasser M et al (2003b) Geduldige Technologie für ungeduldige Patienten: Führt Ubiquitous Computing zu mehr Selbstbestimmung? In: Mattern F (ed) Total vernetzt. Springer, Heidelberg, pp 159-186

Nunamaker J, Chen M, Purdin T (1991) Systems development in information systems research. J Management Information Systems 7(3):89106

Padlipsky MA (1985) The elements of networking style, and other animadversions on the art of intercomputer networking. Prentice Hall

Postel J (1980) DoD standard internet protocol. IETF RFC 760. http://www.ietf.org/rfc/rfc760. txt. Accessed 2008-05-01

Postel J (1985) DoD statement on the NRC report. IETF RFC 945. http://www.ietf.org/rfc/rfc945. txt. Accessed 2008-05-01

Saltzer JH, Reed DP, Clark D (1984) End-to-end arguments in system design. ACM Trans Computer Systems 2(4):277 ff

Schulze G, Müller G (1985) EARN/DFN gateway for message handling. GI-NTG Conference on Communication in Distributed Systems. Berlin

Simon HA (1996) The sciences of the artificial, 3rd ed. MIT Press

Witt M (1986) Moving from DoD to OSI protocols: a first step. Computer Communication Rev 16(2):2-7 